\title{
Use of a Weighted Conflict Graph in the Channel Selection Operation for Wi-Fi Networks
}

\author{
Lafdal Abdelwedoud $^{1,2}$, Anthony Busson ${ }^{1}$ and Isabelle Guérin-Lassous ${ }^{1}$ \\ ${ }^{1}$ University of Lyon, UCBL, EnsL, CNRS, Inria, LIP, Lyon, France \\ Name.Surname@ens-lyon.fr \\ ${ }^{2}$ Univ Nouakchott Al Aasriya, Fac of Science and Technology, Dept of Mathematics and Computer Science, \\ Nouakchott, Mauritanie.
}

\begin{abstract}
Allocation algorithms in IEEE 802.11-based WLAN, that consist to associate to each AP a channel, are mainly based on a conflict graph that represents the conflicts (interference, CCA detection, etc.) between APs. In this paper, we propose to use an enriched version of the conflict graph, namely a weighted conflict graph. This latter models the CCA detection that can be total (all transmissions are detected) or partial. Beside, a model is given to compute the throughput of each AP for a given allocation. This model is combined to a greedy algorithm that aims to find the allocation that maximizes the proportional fairness. Simulations based on the recent IEEE 802.11ax amendment are carried out for small WLANs with a few APs and very dense networks. Results show that when the network is dense or constrained, there is a gain to consider partial detection.

Index Terms-IEEE 802.11, channel allocation, conflict graph.
\end{abstract}

\section{INTRODUCTION}

The management of a Wi-Fi network is a crucial step to provide the best possible quality of experience to users and to efficiently use the network's resources. This step relies, among others, on the network monitoring, the band and channel selection, the users' association to the access points (APs) and the load balancing among the network. Providing an efficient management is still a challenge and a concern for Wi-Fi vendors. Several management operations are often based on the network's conflict graph. Such a conflict graph models the conflicts between devices (APs and/or stations). The conflicts can represent the conflicts in the medium access triggered by the use of the CSMA/CA (Carrier Sense Multiple Access / Collision Avoidance) approach and/or the possible interference between transmitting devices. The conflict graph can be inferred from the network monitoring operation.

In most cases, the conflict graph is considered as a nonweighted graph. It means that two devices are always or never in conflict. We show in [1] that, in practice, this is not the case and that the conflict graph of a Wi-Fi network is partial. It means that two devices may be sometimes in conflict and sometimes not, even though they are static and without a radical change in the environment. Therefore, the use of a non-weighted conflict graph may lead to misleading management operations resulting in a suboptimal use of the network resources.

In this paper, we propose to use a weighted conflict graph representing the partial conflicts between the network's devices in the management operations. We focus our study on the channel selection operation. A large set of channel allocation solutions are based on a non weighted conflict graph like, for instance, in [2]-[5]. Our contributions are twofold: i) we propose a solution that allocates channels to access points knowing the weighted conflict graph between the APs of the network ; ii) we compare the network performance obtained thanks to this allocation with a classical channel allocation using a simple non-weighted conflict graph.

The paper is organized as follows: in Section II, we describe the problem studied in this paper. The proposed channel allocation based on a weighted conflict graph is described in Section III. The simulation results obtained with the proposed allocation are given in Section IV. We also compare our solution with a channel allocation solution based on a classical non weighted conflict graph. We conclude in Section V.

\section{Problem statement}

In this study, we only consider the conflicts between APs. The users' stations are not considered as they can appear and disappear within a short time scale whereas the APs have a more permanent situation in the network. We consider that AP $A$ is in conflict with AP $B$ when AP $A$ can detect, via the CCA (Clear Channel Assessment) mechanism, the transmission of AP $B$ preventing AP $A$ from transmitting. Such a conflict is often modeled by a graph in which the two APs are represented by two nodes in the graph and the conflict with an edge (directed or not) between these two nodes.

Usually the conflict graph is considered as non-weighted. It means that if there is an edge between two nodes then the conflict is considered as permanent between the two considered APs and arising all the time. We have shown in [1] that an AP may sometimes detect the transmission of another AP and may sometimes not even if these two APs are static. This can be explained by the fast fading that can occur on Wi-Fi channels. It means that the conflict is not permanent but partial. Such a partial conflict can be modeled by a weighted conflict graph in which the weight on an edge (directed or not depending if the conflict is considered as asymmetrical or not) represents the fraction of time during which the AP (at one end of the considered edge) senses the activity of the AP (at the other end of the edge) when this latter transmits all the time (in saturation). 
We claim that using a non weighted conflict graph in the management of a Wi-Fi network may lead to a suboptimal use of the network's resources. To support this statement, let's consider a toy example with 3 APs as shown in Fig. 1. In this example, the APs are located such that AP 2 is always in conflict with APs 1 and 3 (the weights of the corresponding edges are 1) and AP 1 and AP 3 have a partial conflict of 0.36 (meaning that they detect each other $36 \%$ of the time).

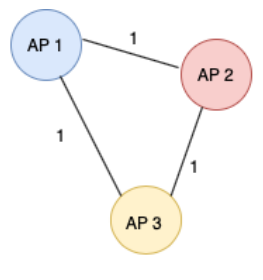

Non weighted conflict graph

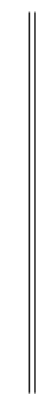

Fig. 1: A toy example for the conflict graph.

Let's assume that only 2 orthogonal channels can be used for this example. When considering the non weighted conflict graph, a classical channel allocation can set APs 1 and 2 on the same channel and AP 3 on the other channel. When considering the weighted conflict graph, the channel allocation obtained with the solution described in Section III sets APs 1 and 3 on the same channel and AP 2 on the other one. Simulations obtained with the ns-3 simulator (the simulation parameters are described in more details hereafter) show that with the second allocation the overall throughput increases with a percentage ranging from $24 \%$ to $12 \%$ as function of the distance between the stations and their AP (from 1 to 17 meters). This is explained by the fact that allocating the same channel to APs with partial detection will enable more transmissions, leading to a better overall throughput. If the partial detection is not modeled in the conflict graph, then the channel allocation solution will not be aware of this possibility and may not boost the use of the same channel on partial links.

\section{MODEL AND CHANNEL ALLOCATION ALGORITHM}

We consider a Wi-Fi network composed of $N$ APs and $n$ stations. We assume that each station is associated to exactly one AP of the network. The variable $x_{k, i}$ indicates if station $k$ is associated to AP $i\left(x_{k, i}=1\right)$ or not $\left(x_{k, i}=0\right)$. A channel allocation is represented through a vector $C$ of size $N$ where the $i^{\text {th }}$ element is the channel number allocated to AP $i$. Our solution is based on an estimation of the throughput that can be obtained by each AP for a given channel allocation.

\section{A. A model to evaluate a channel allocation}

We extend the model proposed in [6] to estimate the throughput of each AP under a given channel allocation. This model relies on a non-weighted conflict graph denoted $S$. The second parameter used by the model is the matrix $\left(r_{k, j}\right)_{k, j}$ where $r_{k, j}$ is the achievable throughput between AP $j$ and station $k$. We define the achievable throughput between AP $j$ and station $k$ as the maximum throughput that is obtained between these two devices in absence of other Wi-Fi nodes. This model assumes that the network is saturated, i.e. an AP has always a frame to send, and that an AP, in average, sends the same number of frames to each of its associated stations.

Our extension consists in introducing the partial detections. The coefficients of the matrix $S$ are substituted by the coefficients $w_{j, i}\left(0 \leq w_{j, i} \leq 1\right)$ that represent the partial CCA detection between APs. In [7], we describe a method to infer the weighted conflict graph of an infrastructure-based Wi-Fi network. The coefficient $w_{j, i}$ depends on the AP location, the radio environment and the channel allocation as two APs using two orthogonal channels do not interfere with each other. The matrix of these weights is denoted $W(C)$. Thus, given a channel allocation and the underlying weighted conflict graph of the network, the mean throughput of AP $i$ is modeled as:

$$
\begin{aligned}
& \text { throughput }\left(A P_{i}, C, W(C)\right)= \\
& \frac{1}{\sum_{k=1}^{n} x_{k, i}} \times \frac{1}{\sum_{j=1}^{N}\left(\frac{w_{j, i}}{\sum_{k=1}^{n} x_{k, j}} \sum_{k=1}^{n} \frac{x_{k, j}}{r_{k, j}}\right)}
\end{aligned}
$$

A channel allocation $C$ can be evaluated through the proportional fairness (PF) metric that offers a good trade-off between throughput and fairness. It is defined as:

$$
P F(C, W(C))=\sum_{i=1}^{N} \ln \left(\text { throughput }\left(A P_{i}, C, W(C)\right)\right)
$$

Our optimization problem aims at finding the channel allocation that maximizes the proportional fairness, given a fixed number of available channels:

$$
\max _{C} P F(C, W(C))
$$

\section{B. A greedy channel allocation}

The solution (Algorithm 1) to the optimization problem defined in Eq. 3 is based on a local search algorithm. It starts from a random channel allocation. At each step, an AP $i$ is selected. For this particular AP, the PF metric is evaluated for each channel. The channel that offers the best PF metric is assigned to this AP (if it was not already assigned). Then, another AP is chosen and so on. When all APs have been selected, the algorithm performs a new round. The algorithm stops when there is no improvement for a whole round.

In terms of complexity, the most costly instruction is the computation of the PF metric which has an upper bound complexity of $O\left(N \cdot n^{2}\right)$. Therefore the overall complexity of the algorithm is $O\left(k .|C| . N^{3} . n\right)$ if $k$ rounds of the algorithm is executed. Note that the complexity of Algorithm 1 is the same when a weighted conflict graph is used as when a non weighted conflict graph is used. 


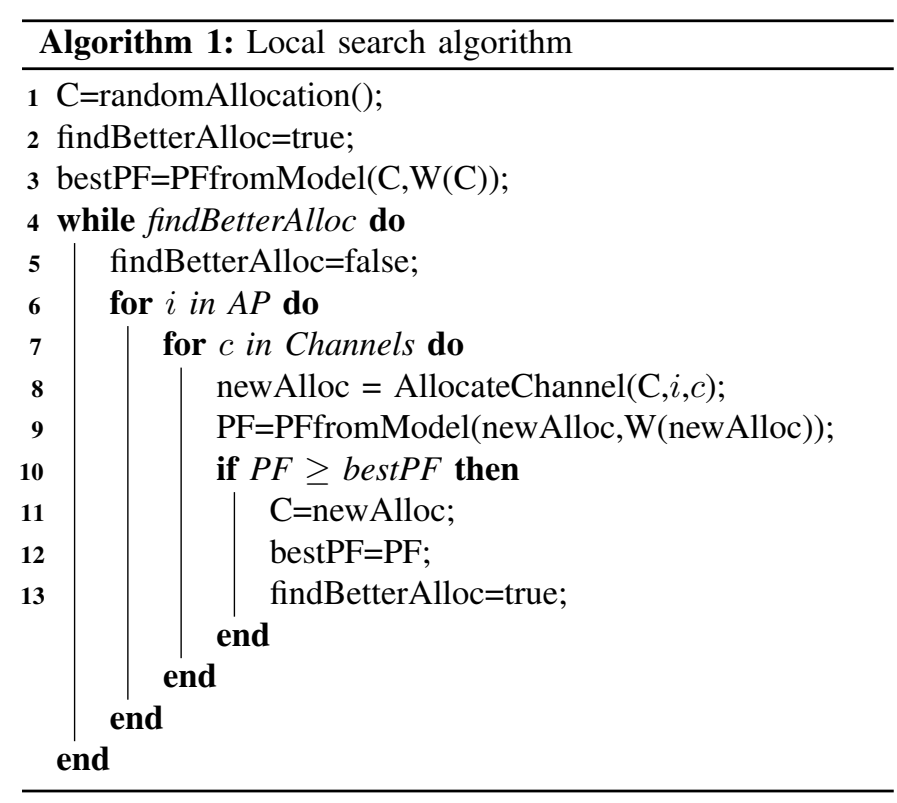

IV. NuMERICAL RESULTS

\begin{tabular}{|c|c|}
\hline Parameters & Value \\
\hline \hline ns-3 version & 3.31 \\
\hline Wi-Fi version & IEEE $802.11 \mathrm{ax}$ \\
\hline Wif-Fi manager & Ideal \\
\hline Propagation model & $\beta \xi \min \left(1, \frac{1}{\text { distance }^{3}}\right)$ \\
\hline$\beta$ & $10^{4.667}$ \\
\hline Fading $(\xi)$ & LogNormal $(0.0,1.0)$ \\
\hline Traffic & Saturated \\
\hline
\end{tabular}

TABLE I: Simulation parameters

\begin{tabular}{|c|c|c|c|c|}
\hline Nb of APs & 4 & 5 & 6 & 7 \\
\hline Throughput gain (in Mb/s) & 108 & 114 & 70 & 72 \\
\hline PF gain (linear scale) & 3,5 & 2 & 1.7 & 2.7 \\
\hline
\end{tabular}

TABLE II: Throughput and PF gains when using the weighted conflict graph

The proposed approach is validated through a large set of realistic simulations. We performed ns-3 simulations to validate i) the accuracy of our model with regard to realistic simulations and ii) to evaluate the gain of using a weighted conflict graph to allocate channels. We changed the physical layer of ns-3.31 by adding a log-normal fading to the propagation model leading to partial detection between 43 and 60 meters. The different simulation parameters are given in Table I. For all the scenarios, we apply Algorithm 1 to perform two allocations: one based on the non weighted conflict graph and the other based on the weighted one. We evaluate the allocations in saturated mode where each AP has always a frame to send.

a) Small topologies: The gain of using a weighted conflict graph can be important for a small topology as it has been shown in Section II. In order to consider more generic topologies, we generate 40 random topologies from 4 to 7 APs distributed in a ball of radius 50 meters, leading to a simulated area of approximately $8000 \mathrm{~m}^{2}$. For these simulations, we use the ISM band and 3 orthogonal channels. For each AP, one associated station downloads data. Table II gives the mean throughput gain, computed as the sum of throughputs over all APs when considering a weighted conflict graph minus the sum of throughputs with a non-weighted conflict graph. The gain is significant as it varies between $70 \mathrm{Mb} / \mathrm{s}$ and $114 \mathrm{Mb} / \mathrm{s}$. Beside, considering weighted conflict graph improves the PF of a factor between 1.67 and 3.5.

b) Dense scenarios: In this section, we focus on a dense Wi-Fi network to explore the limit of the use of weighted conflict graphs. The scenario corresponds to a stadium where the APs cover the bleachers and users of a side of the stadium. In such a scenario, the Wi-Fi network is very dense and each AP covers a limited number of users to ensure a high quality of experience. The APs are located on a $10 \times 6$ grid (60 APs). 10 stations are associated to each AP leading to 600 users downloading data at the same time. The simulated network is an IEEE 802.11ax network in the $5 \mathrm{GHz}$ band.

Figs 2 shows the theoretical and the simulations results when the distance between two adjacent APs in the grid varies. The distances are chosen to have neighbors in the grid detected at $100 \%$ and 2-hop neighbors partially detected. It leads to distances between 10 and 26 meters. For greater distances the conflict graph does not present any partial detection. For small distances, the error in the PF estimation is quite important between the model and the simulations. It is mainly due to the high contention that causes starvation for a few APs. The nature of the PF function makes it very sensitive to small values leading to this error. But as the distance increases, starvation becomes rare and the error decreases significantly. Note that this error is smaller when considering the mean throughput per AP (Fig. 2). In terms of performance, the difference between the two PF values (with and without the weighted conflict graph) varies from 3 to 10 (and thus of several order of magnitude in its linear form). The throughput gain is approximately of $10 \%$ per AP for distances up to 22 meters and is negligible for greater distances. The gain between the two approaches is thus clearly in terms of fairness between APs rather than throughput. We observe the same trend in Fig. 3 when the number of available channels increases. The PF metric is significantly greater when using a weighted conflict graph and when the number of channels if less than 7 (the network is then more constrained). For a greater number of channels, the performance becomes similar for the two allocations.

When the number of channels is low, as for the ISM band, there is thus an important gain to use weighted conflict graph. In the $5 \mathrm{GHz}$ band where there are up to 23 orthogonal channels, there is no real benefit to use a weighted conflict graph. But, if channels are aggregated (40 MHz or $80 \mathrm{MHz}$ ), the allocation is more constrained. For instance, results for the $40 \mathrm{MHz}$ width channel in the $5 \mathrm{GHz}$ band are shown in Table III for 4 channels (UNII-1 and UNII-2 band) and 12 channels (where UNII-2 extended and UNII-3 are added). The table shows that there is a clear benefit to use the weighted 

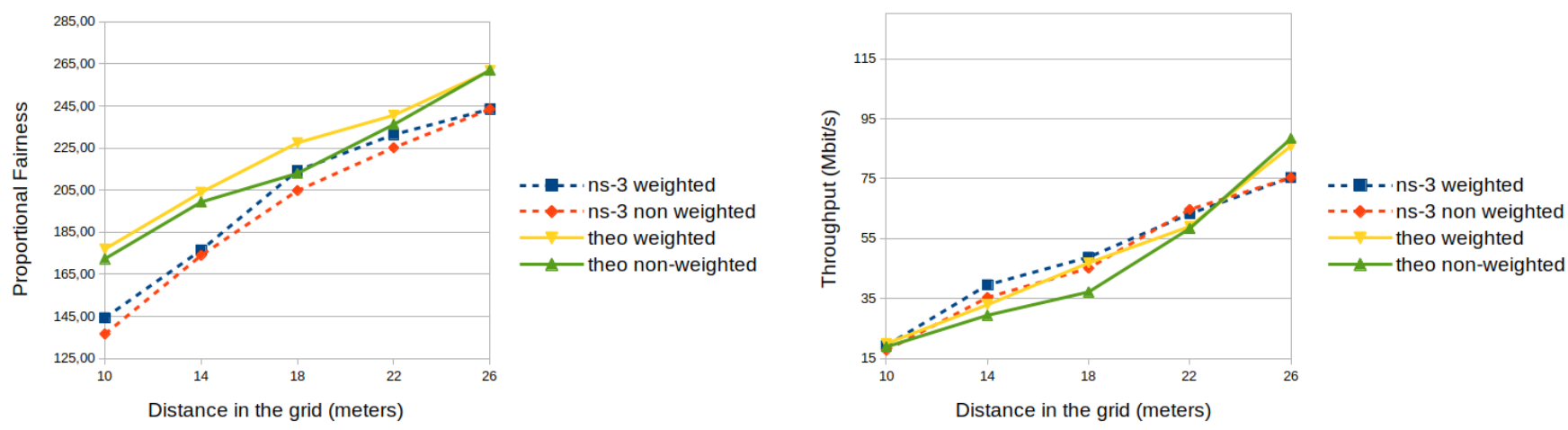

Fig. 2: Comparison of the channel allocation performance with weighted and non-weighted conflict graphs in the $5 \mathrm{GHz}$ band and for $20 \mathrm{MHz}$ channels. The distance between two APs in the grid varies. 3 channels are used.
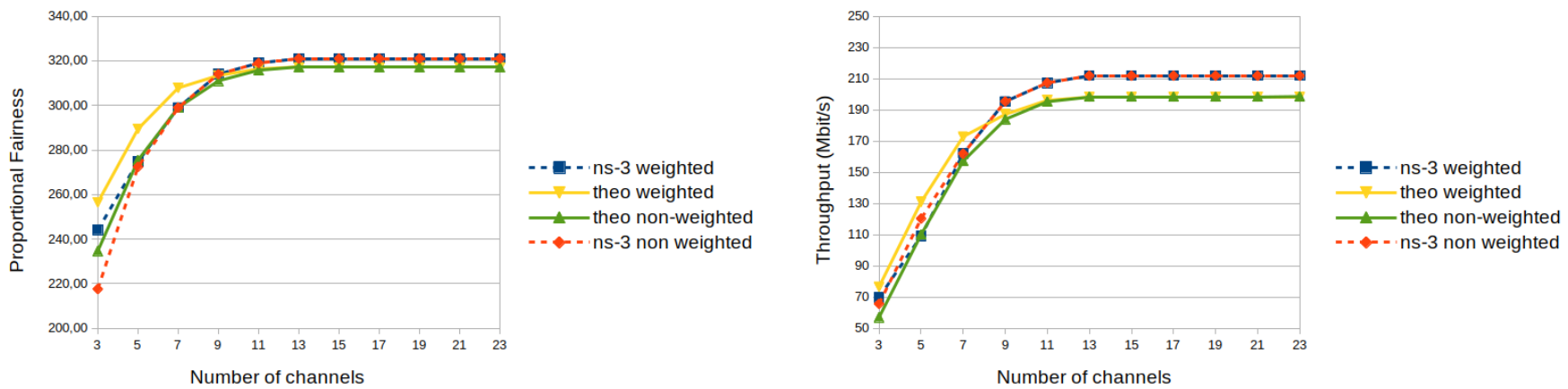

Fig. 3: Comparison of the channel allocation performance with weighted and non-weighted conflict graphs in the $5 \mathrm{GHz}$ band and for $20 \mathrm{MHz}$ channels. The number of channels varies. The distance between two APs in the grid is 25 meters.

\begin{tabular}{|c|c|c|c|c|}
\hline Nb of channels & PF (weighted) & PF (non-weighted) & Throughput (weighted) & Throughput (non-weighted) \\
\hline \hline 4 & 327.2 & 326.1 & 388.7 & 403.4 \\
\hline 12 & 369.5 & 365.3 & 711.9 & 666.7 \\
\hline
\end{tabular}

TABLE III: Simulation results for IEEE $802.11 \mathrm{ax}$ and $40 \mathrm{MHz}$ channel width. The distance between APs in the grid is $27 \mathrm{~m}$.

conflict graph in particular for the 12 channels case.

\section{CONClusion}

Many channel allocation algorithms rely on a conflict graph to model conflicts between APs. In this paper, we propose to use a weighted conflict graph capturing the level of CCA detection between APs and consequently their capacity to share a channel at the same time. We propose a channel allocation algorithm based on a local search approach, a weighted conflict graph and a model estimating the throughput per AP for a given channel allocation. Simulations of a large set of scenarios and using IEEE 802.11ax show that our model is able to accurately estimate objective functions based on throughput. Also, simulation results empirically show that an allocation considering a weighted conflict graph offers better results in terms of proportional fairness for a large set of topologies as soon as the network is dense or constrained. In this case, the algorithm tends to reuse a same channel between APs that partially detect each other.

\section{REFERENCES}

[1] L. Abdewedoud, A. Busson, I. Guérin-Lassous, and M. Foare. A passive method to infer the weighted conflict graph of an ieee 802.11 network. In ADHOC-NOW, pages 1-9, 2019.

[2] J. Riihijarvi, M. Petrova, P. Mahonen, and J. D. A. Barbosa. Performance evaluation of automatic channel assignment mechanism for ieee 802.11 based on graph colouring. In 2006 IEEE 17th International Symposium on Personal, Indoor and Mobile Radio Communications, pages 1-5, 2006.

[3] T. Mahboob, H.Y. Lee, M. Shin, and et al. SDN-Based Centralized Channel Assignment Scheme Using Clustering in Dense WLAN Environments. Wireless Personal Communications, 2020.

[4] K. Nakashima, S. Kamiya, K. Ohtsu, K. Yamamoto, T. Nishio, and M. Morikura. Deep reinforcement learning-based channel allocation for wireless lans with graph convolutional networks. IEEE Access, 8:31823$31834,2020$.

[5] S. Chieochan, E. Hossain, and J. Diamond. Channel assignment schemes for infrastructure-based 802.11 wlans: A survey. IEEE Communications Surveys Tutorials, 12(1):124-136, 2010.

[6] M. Amer, A. Busson, and I. Guérin-Lassous. Association Optimization in Wi-Fi Networks based on the Channel Busy Time Estimation. In IFIP Networking, 2018.

[7] L. Abdelwedoud, A. Busson, I. Guérin-Lassous, M. Foare, M. L. Diakité, and M. F. Nanne. Inference of a clear channel assessment based conflict graph. Internet Technology Letters. 